http://dx.doi.org/10.15407/dopovidi2016.09.007

УДК 517.94

\title{
К.Н. Андреев
}

Физико-технический институт низких температур им. Б. И. Веркина НАН Украины, Харьков

E-mail: kirill.andreev@ukr.net

\section{Интегралы движения уравнения Кортевега-де Фриза в классе решений типа ступеньки}

\section{(Представлено академиком НАН Украины Е.Я. Хрусловым)}

Рассмотрена задача Коши для уравнения Кортевега-де Фриза с начальными данными типа ступенъки. Построены регуляризованные интегралы движсения и получено их представление через данные рассеяния соответствующего уравнения Шредингера.

Ключевые слова: уравнение Кортевега-де Фриза, интегралы движения, данные рассеяния.

Рассмотрим задачу Коши для уравнения Кортевега-де Фриза (КдФ)

$$
\frac{\partial u}{\partial t}-6 u \frac{\partial u}{\partial x}+\frac{\partial^{3} u}{\partial x^{3}}=0,\left.\quad u\right|_{t=0}=u_{0}(x)
$$

с вещественной начальной функцией $u_{0}(x)$ типа ступеньки

$$
\lim _{x \rightarrow-\infty} u_{0}(x)=c^{2}, c>0, \quad \lim _{x \rightarrow+\infty} u_{0}(x)=0 .
$$

В работе [1] доказано, что при соответствующем выборе начальной функции $u_{0}(x)$ задача (1), (2) имеет решения шварцевского типа, обладающие свойствами

$$
\max _{|t| \leq T} \int_{-\infty}^{0}\left(1+|x|^{m}\right)\left|u(x, t)-c^{2}\right| d x<+\infty
$$

(C) К.Н. Андреев, 2016 


$$
\begin{aligned}
& \max _{|t| \leq T} \int_{0}^{+\infty}\left(1+x^{m}\right)|u(x, t)| d x<+\infty, \quad t \geq 0 \\
& \max _{|t| \leq T} \int_{-\infty}^{+\infty}\left(1+|x|^{m}\right)\left|\frac{\partial^{j} u(x, t)}{\partial x^{j}}\right| d x<+\infty, \quad j=1,2 \ldots, \quad m=0,1,2, \ldots,
\end{aligned}
$$

при всех неотрицательных значениях $T$.

В работе [2] получено, что для решений $u(x, t)$ задачи $(1),(2)$, обладающих свойствами $(3)$, существует бесконечная серия регуляризованных интегралов движения $J_{j}[u]$, $j=1,2,3, \ldots$. Первые три из них имеют вид

$$
\begin{aligned}
J_{1}[u] & =\int_{-\infty}^{0}\left(-u^{2}(\xi, t)+\frac{4}{3} c^{2} u(\xi, t)-\frac{1}{3} c^{4}\right) d \xi+ \\
& +\int_{0}^{\infty}\left(-u^{2}(\xi, t)+\frac{4}{3} c^{2} u(\xi, t)\right) d \xi \\
J_{2}[u] & =\int_{-\infty}^{0}\left(2 u^{3}(\xi, t)-3 c^{4} u(\xi, t)+u_{\xi}^{2}(\xi, t)+c^{6}\right) d \xi+ \\
+ & \int_{0}^{\infty}\left(2 u^{3}(\xi, t)-3 c^{4} u(\xi, t)+u_{\xi}^{2}(\xi, t)\right) d \xi \\
J_{3}[u] & =\int_{-\infty}^{0}\left(-5 u^{4}(\xi, t)-10 u(\xi, t) u_{\xi}^{2}(\xi, t)+8 c^{6} u(\xi, t)-u_{\xi \xi}^{2}(\xi, t)-3 c^{8}\right) d \xi+ \\
& +\int_{0}^{\infty}\left(-5 u^{4}(\xi, t)-10 u(\xi, t) u_{\xi}^{2}(\xi, t)+8 c^{6} u(\xi, t)-u_{\xi \xi}^{2}(\xi, t)\right) d \xi .
\end{aligned}
$$

При этом $J_{2}[u]$ играет роль гамильтониана, т.е. уравнение КдФ представимо в гамильтоновом виде

$$
u_{t}=\frac{d}{d x} \frac{\delta J_{2}[u]}{\delta u}
$$

где $\frac{\delta}{\delta u}-$ производная Фреше.

Цель настоящей работы - выразить интегралы движения через данные рассеяния оператора Шредингера с потенциалом типа ступеньки.

Как известно [3], уравнение КдФ эквивалентно в пространстве $H^{5}(\mathbb{R})$ уравнению Лакса $\partial_{t} L(t)=[P(t), L(t)]$, где

$$
\begin{aligned}
& L(t)=-\partial_{x}^{2}+u(x, t), \\
& P(t)=-4 \partial_{x}^{3}+6 u(x, t) \partial_{x}+3 u_{x}(x, t) .
\end{aligned}
$$


Приведем необходимые сведения теории рассеяния для оператора Шредингера $L(t)$ на всей оси с потенциалом типа ступеньки. Рассмотрим спектральное уравнение

$$
(L(t) y)(x)=k^{2} y(x), \quad k \in \overline{\mathbb{C}^{+}}, \quad x \in \mathbb{R},
$$

где $u(\cdot, t) \in L_{\text {loc }}^{1}(\mathbb{R})$ и

$$
\lim _{x \rightarrow-\infty} u(x, t)=c^{2}, \quad \lim _{x \rightarrow+\infty} u(x, t)=0 .
$$

Такая задача рассеяния впервые исследовалась в [4], а также [5]. Полное решение этой задачи можно найти в [6]. Предположим, что потенциал $u(x, t)$ достаточно быстро стремится к своим пределам, так что выполнено условие

$$
\left.\int_{0}^{\infty}(1+|x|)|u(x, t)|+\left|u(-x, t)-c^{2}\right|\right) d x<\infty, \quad \forall t \in \mathbb{R} .
$$

Тогда справедливы следующие факты.

1. Уравнение (5) имеет два решения Йоста $\varphi(k, x, t)$ и $\varphi_{1}(k, x, t)$ с асимптотиками

$$
\begin{aligned}
& \lim _{x \rightarrow+\infty} \varphi(k, x, t) e^{-\mathrm{i} k x}=1, \quad \operatorname{Im} k \geqslant 0, \\
& \lim _{x \rightarrow-\infty} \varphi_{1}(k, x, t) e^{\mathrm{i} k_{1} x}=1, \quad \operatorname{Im} k_{1} \geqslant 0,
\end{aligned}
$$

где $k_{1}=\sqrt{k^{2}-c^{2}}-$ спектральный параметр. Эти решения удовлетворяют соотношению рассеяния

$$
T(k, t) \varphi_{1}(k, x, t)=\overline{\varphi(k, x, t)}+R(k, t) \varphi(k, x, t), \quad k \in \mathbb{R},
$$

где $T(k, t), R(k, t)$ - правые коэффициенты прохождения и отражения.

2. Спектр оператора (4) состоит из абсолютно непрерывной части $\mathbb{R}_{+}$и конечного числа отрицательных собственных значений $-\varkappa_{1}^{2}<\ldots<-\varkappa_{m}^{2}<0$. Непрерывный спектр состоит из однократного $\left[0, c^{2}\right]$ и двукратного $\left[c^{2} ; \infty\right)$. В терминах переменных $k, k_{1}$ непрерывный спектр соответствует значениям спектрального параметра $k \in \mathbb{R}$, а двукратный спектр значениям $k_{1} \in \mathbb{R}$.

3. Вронскиан $W(k, t)=\varphi_{1}(k, x, t) \varphi^{\prime}(k, x, t)-\varphi_{1}^{\prime}(k, x, t) \varphi(k, x, t)$ имеет простые нули в точках іж. Кроме этого, единственно возможный нуль может быть в точке $k=0$. Этот случай называется резонансом.

4. Решения $\phi\left(\mathrm{i} \varkappa_{l}, x, t\right)$ и $\phi_{1}\left(\mathrm{i} \varkappa_{l}, x, t\right)$ являются линейно зависимыми собственными функциями оператора (5). Соответствующие нормирующие константы

$$
m_{l}=\left(\int_{\mathbb{R}} \phi^{2}\left(\varkappa_{l}, x, t\right) d x\right)^{-1}, m_{l, 1}=\left(\int_{\mathbb{R}} \phi_{1}^{2}\left(\varkappa_{l}, x, t\right) d x\right)^{-1} .
$$

5. Справедливо следующее тождество:

$$
1-|R(k, t)|^{2}=\frac{k_{1}}{k}|T(k, t)|^{2}, \quad|k|>c .
$$

6. Величина $|T(k, t)|$ не зависит от $t$ при $k_{1} \in \mathbb{R}[7]$, а при $k \in[-c ; c]$

$\arg T(k, t)=\arg T(k, 0)-4 k^{3} t$. 
Кроме того, $m_{l}(t)=m_{l}(0) e^{-8 \varkappa_{l}^{3} t}$.

7. Решение $u(x, t)$ начальной задачи (1)-(3) может быть однозначно восстановлено по правым данным рассеяния $\left\{R(k, t), \mathrm{i} \varkappa, m_{l}, l=\overline{1, m}\right\}$.

8. Если $u(\cdot, t) \in C^{n}(\mathbb{R})$, то $R(k, t)=O\left(\frac{1}{k^{n+1}}\right), k \rightarrow \infty$.

Рассмотрим подробнее мероморфную функцию $T(k, t)$. Зная ее полюсы і,$l=\overline{1, m}$, в верхней полуплоскости можно определить функцию $A(k, t)$, которая аналитична при $\operatorname{Im} k>0$ и не имеет там нулей:

$$
A(k, t)=\frac{\mathrm{i}}{k_{1}} \log \left[\prod_{l=1}^{m} \frac{k-\mathrm{i} \varkappa_{l}}{k+\mathrm{i} \varkappa_{l}} T(k, t) \sqrt{\frac{k_{1}}{k}}\right] .
$$

При $\operatorname{Im} k>0$ по теореме Коши справедливо интегральное представление через мнимую часть

$$
A(k, t)=\frac{1}{\pi} \int_{-\infty}^{+\infty} \frac{\operatorname{Im} A(s, t)}{s-k} d s
$$

откуда, используя связь (6) и $(7)$ коэффициентов прохождения $T(k, t)$ и отражения $R(k, t)$, получаем представление подынтегральной функции $\operatorname{Im} A(k, t)$ в виде

$$
\operatorname{Im} A(k, t)=\left\{\begin{array}{l}
\frac{1}{2 \sqrt[t]{k^{2}-c^{2}}} \log \left(1-|R(k, t)|^{2}\right), \quad k \in \mathbb{R} \backslash[-c ; c], \\
\frac{1}{\sqrt[t]{c^{2}-k^{2}}}\left[\frac{1}{2} \arg R(k, t)+\arg \sqrt{\frac{k_{1}}{k}}+\sum_{l=1}^{m} \arg \frac{k-\mathrm{i} \varkappa_{l}}{k+\mathrm{i} \varkappa_{l}}\right], \quad k \in[-c ; c],
\end{array}\right.
$$

где $\sqrt[+]{ }$ - арифметический квадратный корень.

При $\operatorname{Im} k=0$ доопределяем функцию $A(k, t)$ как предел

$$
\left.A(k, t)\right|_{\operatorname{Im} k=0}=\left.\lim _{\operatorname{Im} k \rightarrow+0} A(k, t)\right|_{\operatorname{Im} k>0} .
$$

Тогда

$$
T(k, t)=\sqrt{\frac{k}{k_{1}}} \prod_{l=1}^{m} \frac{k+\mathrm{i} \varkappa_{l}}{k-\mathrm{i} \varkappa_{l}} \exp \left\{-\mathrm{i} k_{1} A(k, t)\right\} .
$$

Подставляя (8) в (10) и используя представление для мнимой части (9), получаем интегральное представление

$$
\begin{aligned}
& \log T^{-1}(k, t)=-\frac{i \sqrt{1-\frac{c^{2}}{k^{2}}}}{2 \pi} \int_{\mathbb{R} \backslash[-c ; c]} \frac{\log \left(1-|R(s, t)|^{2}\right)}{\sqrt{s^{2}-c^{2}}} \frac{d s}{1-\frac{s}{k}}- \\
& -\frac{i \sqrt{1-\frac{c^{2}}{k^{2}}}}{\pi} \int_{-c}^{c}\left[\frac{1}{2} \arg R(s, t)+\arg \sqrt{\frac{\sqrt{s^{2}-c^{2}}}{s}}+\sum_{l=1}^{m} \arg \frac{s-\mathrm{i} \varkappa_{l}}{s+\mathrm{i} \varkappa_{l}}\right] \frac{1}{\sqrt{c^{2}-s^{2}}} \frac{d s}{1-\frac{s}{k}}+ \\
& +\sum_{l=1}^{m}\left(\log \left(1-\frac{\mathrm{i} \varkappa_{l}}{k}\right)-\log \left(1+\frac{\mathrm{i} \varkappa_{l}}{k}\right)\right)+\log \sqrt{\frac{k_{1}}{k}} .
\end{aligned}
$$


Учитывая эволюцию данных рассеяния (7) и связь коэффициентов отражения и прохождения (6), находим коэффициенты $c_{n}$ разложения функции $\log T^{-1}(k, t)$ в ряд по степеням $(-2 \mathrm{i} k)^{n}:$

$\log T^{-1}(k, t)=\sum_{n=1}^{\infty} \frac{c_{n}(t)}{(-2 \mathrm{i})^{n}} \frac{1}{k^{n}}$.

Используя разложения Тейлора для $\sqrt{1-\frac{c^{2}}{k^{2}}} \frac{1}{1-(s / k)}$ и для $\log \left(1 \pm\left(\mathrm{i} \varkappa_{l} / k\right)\right)$ по степеням $(-2 \mathrm{i} k)^{n}$ и учитывая, что $\operatorname{Im} A(k)$ нечетно при $k \in[-c ; c]$, получаем при $j=1,2, \ldots$

$$
\begin{aligned}
& c_{2 j-1}(t)=\frac{1}{\pi} \int_{\mathbb{R} \backslash[-c ; c]} \log \left(1-|R(s, t)|^{2}\right) \frac{(-1)^{j-1} 2^{2 j-2} d_{2 j-1}(s)}{\sqrt{s^{2}-c^{2}}} d s+ \\
& +\frac{1}{\pi} \int_{-c}^{c}\left(\frac{1}{2} \arg R(s, t)+\arg \sqrt{\frac{\sqrt{s^{2}-c^{2}}}{s}}+\sum_{l=1}^{m} \arg \frac{s-\mathrm{i} \varkappa_{l}}{s+\mathrm{i} \varkappa l}\right) \frac{(-1)^{j-1} 2^{2 j-1} d_{2 j-1}(s)}{\sqrt{c^{2}-s^{2}}} d s- \\
& \quad-\frac{2^{2 j}}{2 j-1} \sum_{l=1}^{m} \varkappa_{l}^{2 j-1}, \\
& c_{2 j}=\frac{(-1)^{j-1}(2 c)^{2 j}}{4 j} .
\end{aligned}
$$

где коэффициенты $d_{2 j-1}(s), e_{2 j-1, l}$ при $j=1,2, \ldots$ можно найти исходя из соотношений

$$
d_{2 j-1}(s)=a_{1}(s) b_{j}+\ldots+a_{j}(s) b_{1},
$$

а $a_{p}(s), b_{p}, p=\overline{1, j}$, находятся исходя из

$$
a_{p}(s)=s^{2 p-1}, \quad b_{p}=\frac{(2 p-2) !}{(3-2 p)(p-1) ! 4^{p-1}} c^{2(p-1)} .
$$

Первые три коэффициента при соответствующих степенях выглядят следующим образом:

$$
\begin{aligned}
& \frac{1}{k^{1}}: d_{1}(s)=s, \\
& \frac{1}{k^{3}}: d_{3}(s)=s^{3}-\frac{c^{2}}{2} s, \\
& \frac{1}{k^{5}}: d_{5}(s)=s^{5}-\frac{c^{2}}{2} s^{3}-\frac{c^{4}}{8} s,
\end{aligned}
$$

Отсюда следует, что можно выразить регуляризованные интегралы движения для постоянного фона через данные рассеяния. Первые два из них имеют вид:

$$
J_{1}[u]=c_{3}(t)+\frac{4}{3} c^{2} c_{1}(t)=\frac{1}{\pi} \int_{\mathbb{R} \backslash[-c ; c]} \log \left(1-|R(s, t)|^{2}\right) \frac{-4 s^{3}+\frac{10}{3} c^{2} s}{\sqrt{s^{2}-c^{2}}} d s+
$$




$$
\begin{aligned}
& +\frac{1}{\pi} \int_{-c}^{c}\left(\frac{1}{2} \arg R(s, t)+\arg \sqrt{\frac{\sqrt{s^{2}-c^{2}}}{s}}+\sum_{l=1}^{m} \arg \frac{s-\mathrm{i} \varkappa_{l}}{s+\mathrm{i} \varkappa_{l}}\right) \frac{-8 s^{3}+\frac{20}{3} c^{2} s}{\sqrt{c^{2}-s^{2}}} d s+ \\
& +\sum_{l=1}^{m}\left(-\frac{16}{3} \varkappa_{l}^{3}+\frac{16}{3} c^{2} \varkappa_{l}\right), \\
& J_{2}[u]=c_{5}(t)-3 c^{4} \cdot c_{1}(t)=\frac{1}{\pi} \int_{\mathbb{R} \backslash[-c ; c]} \log \left(1-|R(s, t)|^{2}\right) \frac{16 s^{5}-8 c^{2} s^{3}-5 c^{4} s}{\sqrt{s^{2}-c^{2}} d s+} \\
& +\frac{1}{\pi} \int_{-c}^{c}\left(\frac{1}{2} \arg R(s, t)+\arg \sqrt{\frac{\sqrt{s^{2}-c^{2}}}{s}}+\sum_{l=1}^{m} \arg \frac{s-\mathrm{i} \varkappa_{l}}{s+\mathrm{i} \varkappa_{l}}\right) \frac{32 s^{5}-16 c^{2} s^{3}-10 c^{4} s}{\sqrt{c^{2}-s^{2}}} d s+ \\
& +\sum_{l=1}^{m}\left(-\frac{64}{5} \varkappa_{l}^{5}+9 c^{4} \varkappa_{l}\right) .
\end{aligned}
$$

В частном случае, при $c=0$, получаем результат работы [8].

\section{Цитированная литература}

1. Egorova I., Teshl G. On the Cauchy Problem for the Korteweg-de Vries Equation with Steplike Finite-Gap Initial Data II. Perturbations with Finite Moments // J. Anal. Math. - 2011. - 115, No 1. - P. 71-101.

2. Андреев K.Н., Хруслов Е.Я. Регуляризованные интегралы движения уравнения Кортевега-де Фриза в классе неубывающих функций // Укр. мат. журн. - 2015. - 67, № 12. C. $1587-1601$.

3. Lax P.D. Integrals of nonlinear equations and solitary waves // Comm. Pure Appl. Math. 1968.- 21, No 2. - C. 467-490.

4. Буслаев В.С., Фомин В.Н. К обратной задаче рассеяния для одномерного уравнения Шредингера на всей оси // Вестн. Ленингр. ун-та. - 1962. - 17, № 1. - С. 56-64.

5. Cohen A., Kappeler T. Scattering and inverse scattering for steplike potentials in the Schrödinger equation // Indiana Univ. Math. J. - 1985. - 34. - P. 127-180.

6. Egorova I., Gladka Z., Lange T.-L., Teschl G. Inverse scattering theory for Schrödinger operators with steplike potentials // J. Math. Phys., Anal., Geom. - 2015. - 11. - P. 123-158.

7. Хруслов Е. Я. Асимптотика решения задачи Коши для уравнения Кортевега-де Фриза с начальными данными типа ступеньки // Матем. сб. - 1976. - 99, № 2. - С. 261-281.

8. Захаров В.Е., Фаддеев Л.Д. Уравнение Кортевега-де Фриса - вполне интегрируемая гамильтонова система // Функц. анализ и его прил. - 1971. - 5, вып. 4. - С. 18-27.

\section{References}

1. Egorova I., Teshl G. J. Anal. Math., 2011, 115: No 1: 71-101.

2. Andreev K. N., Khruslov E. Ya. Ukr. Mat. Zh., 2015, 67: No 12: 1587-1601 (in Russian).

3. Lax P. D. Comm. Pure Appl. Math., 1968, 21: No 2: 467-490.

4. Buslaev V.S., Fomin V.N. Vestn. Leningr. Univ., 1962, 17: No 1: 56-64 (in Russian).

5. Cohen A., Kappeler T. Indiana Univ. Math. J., 1985, 34: 127-180.

6. Egorova I., Gladka Z., Lange T.-L., Teshl G. J. Math. Phys., Anal., Geom., 2015, 11: 123-158.

7. Hruslov E.J. Math. USSR Sb., 1976, 28: No 2: 229-248.

8. Zakharov V.E., Faddeev L.D. Funct. Anal. Appl., 1971, 5: No 4: 280-287. 


\section{К.М. Андреєв}

Фізико-технічний інститут низьких температур ім. Б.І. Вєркіна НАН України, Харків E-mail: kirill.andreev@ukr.net

\section{Інтеграли руху рівняння Кортевега-де Фріза в класі розв'язків типу сходинки}

Розглянуто задачу Коші для рівняння Кортевега-де Фріза з початковими даними типу сходинки. Побудовано регуляризовані інтеграли руху та отримано їх вираз через дані розсіювання відповідного рівняння Шредінгера.

Ключові слова: рівняння Кортевега-де Фріза, інтеграли руху, дані розсіювання.

\section{K.M. Andreiev}

B. I. Verkin Institute for Low Temperature Physics and Engineering of the NAS of Ukraine, Kharkiv

E-mail: kirill.andreev@ukr.net

\section{Integrals of motion of the Korteweg-de Vries equation in a class of steplike solutions}

We study the Cauchy problem for the Korteweg-de Vries with steplike initial data. The regilarized integrals of motion are constructed and expressed via associated scattering data of the Schrödinger equation.

Keywords: Korteweg-de Vries equation, integrals of motion, scattering data. 